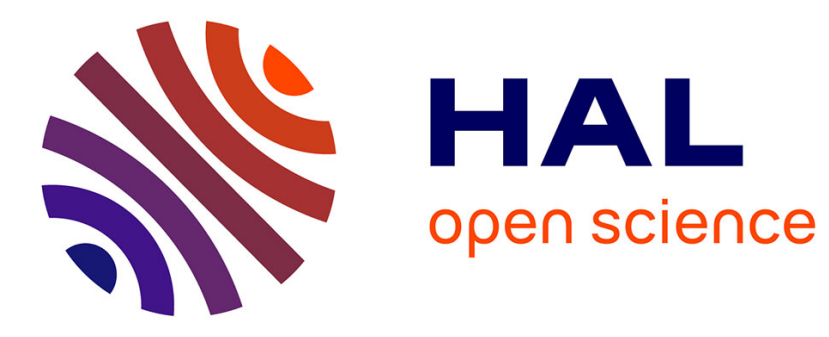

\title{
Msx1 and Msx2 in limb mesenchyme modulate digit number and identity.
}

Vardina Bensoussan-Trigano, Yvan Lallemand, Cécile Saint Cloment, Benoît Robert

\section{- To cite this version:}

Vardina Bensoussan-Trigano, Yvan Lallemand, Cécile Saint Cloment, Benoît Robert. Msx1 and Msx2 in limb mesenchyme modulate digit number and identity.. Developmental Dynamics, 2011, 240 (5), pp.1190-202. 10.1002/dvdy.22619 . pasteur-01120651

\section{HAL Id: pasteur-01120651}

\section{https://hal-pasteur.archives-ouvertes.fr/pasteur-01120651}

Submitted on 26 Feb 2015

HAL is a multi-disciplinary open access archive for the deposit and dissemination of scientific research documents, whether they are published or not. The documents may come from teaching and research institutions in France or abroad, or from public or private research centers.
L'archive ouverte pluridisciplinaire HAL, est destinée au dépôt et à la diffusion de documents scientifiques de niveau recherche, publiés ou non, émanant des établissements d'enseignement et de recherche français ou étrangers, des laboratoires publics ou privés.

\section{(ㅇ)(1) $\$$}

Distributed under a Creative Commons Attribution - NonCommercial - NoDerivatives| 4.0 


\section{Msx1 and Msx2 in limb mesenchyme modulate digit number and identity}

Vardina Bensoussan-Trigano $^{1 *}$, Yvan Lallemand ${ }^{1}$, Cécile Saint Cloment and Benoît Robert ${ }^{\S}$ Institut Pasteur, Génétique Moléculaire de la Morphogenèse, CNRS URA 2578 25 rue du Dr Roux, 75724 Paris Cedex15, France

${ }^{1}$ These authors contributed equally to this work *Present address: Department of Biological Regulation, Weizmann Institute of Science, Rehovot 76100, Israel.

§ Corresponding author (Tel: 331456889 65; Fax: 331456889 63; e-mail: benoit.robert@pasteur.fr)

Running title: $M s x 1$ and $M s x 2$ in limb mesenchyme

Key words : Msx, conditional mutation, limb, patterning, mesenchyme, Shh, anteroposterior axis

Grant sponsor: Agence Nationale de la Recherche; Grant number: ANR 06 MRAR027 02 


\section{ABSTRACT}

Msx1 and Msx2 encode homeodomain transcription factors that play a crucial role in limb development. However, the limb phenotype of the double $M s x 1^{\text {null/null }} M s \times 2^{\text {null/null }}$ mutant is difficult to analyze, particularly along the antero-posterior axis, because of the complex effects of the double mutation on both ectoderm- and mesoderm-derived structures. Namely, in the mutant, formation of the apical ectodermal ridge (AER) is impaired anteriorly and, consequently, the subjacent mesenchyme does not form. Using the Cre/loxP system, we investigated the respective roles of Msx genes in ectoderm and mesoderm by generating conditional mutant embryos with no Msx activity solely in the mesoderm. In these mutants, the integrity of the ectoderm-derived AER was maintained, allowing formation of the anterior mesenchyme. With this strategy, we demonstrate that mesenchymal expression of Msxl and $M s \times 2$ is required for proper Shh and Bmp4 signaling to specify digit number and identity.

\section{INTRODUCTION}

The Msx genes of vertebrates constitute a small family of homeobox-containing genes encoding transcription factors. During mouse development, Msx genes are expressed in a wide range of tissues and in several organs used as models for studying pattern formation and tissue interactions. These include neural crest, branchial arches, cranial sensory placodes, hair follicles, mammary glands, teeth and limbs (Chen et al., 1996; reviewed in Davidson, 1995; Bendall and Abate-Shen, 2000). However, because of functional redundancy between Msxl and $M s \times 2$, null mutants for either gene display limited developmental defects as compared to the Msx expression patterns (Satokata and Maas, 1994; Satokata et al., 2000). Accordingly, the role of Msx genes in several embryological fields can be studied only in the MsxI $I^{\text {null/null }}$ $M s x 2^{\text {null/null }}$ double homozygous mutant. In particular, limb morphology is profoundly altered only when both Msxl and Msx2 are mutated (Lallemand et al., 2005). However, as both genes 
are expressed in the limb mesoderm and ectoderm, the phenotype may result from defects in either germ layer, making it difficult to analyze. Ecto-mesodermal interactions are crucial for limb formation. At early stages (i.e. before 9.75 days post-coitum (dpc) in the mouse embryo), dorso-ventral polarity is transferred from mesoderm to ectoderm (Altabef et al., 1997; Michaud et al., 1997) by a process that may involve BMP signaling (Ahn et al., 2001; reviewed in Robert, 2007). This transfer is requested for the formation of the apical ectodermal ridge (AER), a thickened row of ectodermal cells at the interface between the limb bud dorsal and ventral domains. The AER, in turn produces growth factors of the Fibroblast growth factor (Fgf) family that promote limb outgrowth along its proximo-distal axis (from the shoulder or hip to the extremity of the digits) via a regulatory loop between AER Fgfs (mainly Fgf8 and Fgf4) and Fgf10 that is produced in the mesenchyme (Xu et al., 1998). $F g f 4, F g f 8, F g f 9$ and $F g f 17$ are expressed specifically in the mouse AER. Fgf8 expression is initiated in prospective AER cells of the nascent limb bud and, subsequently, expressed throughout the AER until it regresses (Crossley and Martin, 1995). By contrast, Fgf4, Fgf9 and Fgfl7 expression is initiated only after the AER is formed, is restricted to the posterior AER and is downregulated before AER regression (Sun et al., 2000).

In the Msx1 Msx2 double null mutant, dorso-ventral information transfer is altered at the anterior of the limb bud and the anterior third of the AER does not develop, which results in agenesis of the anteriormost part of the limb mesenchyme, including the presumptive territory of digit 1 (thumb or big toe) (Lallemand et al., 2005). This precludes further analysis using genetic markers expressed in a polarized manner along the anteroposterior (AP) axis (from thumb to pinky). For example, an anterior specific marker like $\operatorname{Pax} 9$ cannot be studied as the region corresponding to its expression domain disappears early in the Msx 1 Msx2 double null mutant limb. Conversely, expression of posterior specific markers such as Hand2 or genes of 
the Hoxd complex (Hoxd11, Hoxd12) appear artificially expanded anteriorly because of the absence of the anterior non-expressing domain.

For these reasons, producing mutant animals in which Msx activity would be eliminated specifically from limb mesenchyme was a requirement. To achieve this goal, we made use of the Msx $2^{\text {Flox-GFP }}$ conditional allele we recently described (Bensoussan et al., 2008) and the Prxl-Cre transgene that drives Cre expression specifically in the limb bud mesenchyme (Logan et al., 2002), in conjunction with an Msxi ${ }^{\text {LacZ }}$ null allele (Houzelstein et al., 1997). Analysis of the Prxl-Cre Msxl Msx2 compound mutants revealed a crucial role for mesodermal expression of $M s x 1$ and $M s x 2$ in specification of digit number and identity along the anteroposterior axis, via their involvement in both Shh and BMP signaling pathways.

\section{RESULTS}

\section{Generation of Prx1-Cre Msx1 Msx2 compound mutants with specific elimination of all}

\section{Msx activity in the limb bud mesoderm}

To investigate Msx function specifically in the limb bud mesoderm, we first combined the conditional floxed allele $M s x 2^{\text {Flox-GFP }}$ (hereafter termed $M s x 2^{\text {Flox }}$ ) and its deleted form, $M s x 2^{\text {null-GFP }}$ (hereafter termed Msx $2^{\text {null }}$ ) (Bensoussan et al., 2008), together with the Prxl-Cre deleter transgene (Logan et al., 2002). The latter directs high-level production of Cre in the limb bud mesenchyme, starting as early as $9.5 \mathrm{dpc}$ in the forelimb and $10.5 \mathrm{dpc}$ in the hindlimb. The efficiency of this strategy to specifically inactivate $M s \times 2$ in the limb bud mesoderm was evaluated by generating Prx1-Cre Msx $2^{\text {null/Flox }}$ embryos and monitoring Msx2 expression by in situ hybridization (ISH). As illustrated (Supplementary Fig. S1A,B), Msx2 expression in the AER was similar in control (Prxl-Cre Msx $2^{+/ F l o x}$ ) and mutant (PrxlCre Msx $2^{\text {null/Flox }}$ ) embryos at $10.5 \mathrm{dpc}$. On the contrary, no Msx2 transcripts were detectable in 
the mutant mesenchyme, thus demonstrating the efficiency of this strategy to delete the $M s \times 2^{\text {Flox }}$ conditional mutant allele specifically in the limb bud mesenchyme.

We further combined the mutant $M s x I^{\text {LacZ }}$ allele (hereafter termed $M s x I^{\text {null }}$ ) (Houzelstein et al., 1997) with the two Msx2 alleles and the Prxl-Cre transgene described above, in order to generate Prx1-Cre Msx $1^{\text {null/null }} M s x 2^{\text {null/Flox }}$ embryos. These lack all Msx activity in the limb bud mesoderm but retain some Msx activity (a single Msx2 functional allele) in the AER. The mating protocol used to produce these mutants (referred to from hereon as Msx conditional double mutants) is described in the supporting information (Supplementary Fig. S1C).

Embryos with a single $M s x 2$ functional allele $\left(M s x 1^{\text {null/null }} M s x 2^{+/ n u l l}\right)$ display minor abnormalities in the limbs.

The Msx conditional double mutants retain a single Msx2 functional allele in the AER (Supplementary Fig. S1C). We have previously shown that hindlimbs from mutants with such a reduced Msx gene dosage in ectoderm and mesoderm (i.e. $M s x 1^{\text {null/null }} M s \times 2^{+/ n u l l}$ mutants) display AP abnormalities (Lallemand et al., 2009). To avoid any misinterpretation of the Msx conditional double mutant phenotype, we carefully reinvestigated the limb phenotype of the $M s x 1^{\text {null/null }} M s \times 2^{+/ n u l l}$ compound mutants. In accordance with our previous results (Lallemand et al., 2009), hindlimbs displayed a slight anterior overgrowth of the mesenchyme at 11.75 dpc that became prominent at $12.5 \mathrm{dpc}$, whereas no such deformation was observed in forelimbs (Fig. 1A-D'). At birth, digit 1 in the mutant hindlimb bore three phalanges instead of two in about $70 \%$ of the animals. In the forelimb, the size and the number of phalanges of digit 1 remained normal. However, the falciform bone was fused with the thumb (Fig. 1I,I'). In keeping with these phenotypic observations, genes normally expressed posteriorly, such as Hoxd genes, systematically displayed anteriorized expression in the hindlimbs, but not forelimbs, of the $M s x I^{\text {null/null }} M s x 2^{+/ n u l}$ embryos (Fig. 1A-D'). Similarly, genes expressed 
anteriorly $(\operatorname{Pax} 9, D l x 5)$ were downregulated in the presumptive digit 1 domain of the mutant hindlimbs, but not in the corresponding region of the forelimbs where $D l x 5$ was unaffected and Pax9 only slightly downregulated (Fig. 1E-H'). In contrast, expression of Shh and Gli3, as well as Glil and Ptcl, two direct targets of Shh signaling, appeared normal in the hindlimbs of these compound mutants (Fig. 1J-L' and data not shown). In conclusion, reducing Msx gene dosage to a single $M s x 2$ functional allele leads to impairment of the AP polarity in the hindlimb, in which the anteriormost region partially loses its specific identity, whereas malformations of the forelimbs are limited to a partial fusion of the falciform bone with digit 1 , with no concomitant detectable alteration in gene expression. These defects must be taken into consideration when analyzing the phenotype of the Prxl-Cre Msx $1^{\text {null/null }}$ Msx $2^{\text {nul/Flox }}$ conditional double mutants.

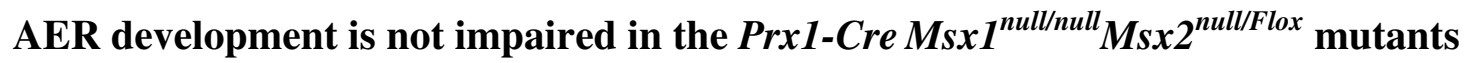

In the double $M s x^{\text {null/null }} M s x 2^{\text {null/null }}$ mutants, the AER does not develop anteriorly during the early steps of limb formation, which results in agenesis of the underlying mesoderm and anterior truncation of the limb. To assess AER formation in the Msx conditional double mutant embryos, we used $F g f 8$ as a read-out of AER activity and morphology (Fig. 2A). Because of specific properties of the Prxl-Cre transgene (see below), we focused our analysis to the forelimbs. At $11.5 \mathrm{dpc}$, in the double $M s x^{\text {null/null }} M s x 2^{\text {null/null }}$ mutant, the anterior third of the AER was missing (Fig. 2C), as previously described (Lallemand et al., 2005). On the contrary, in the Msx conditional double mutant, the AER remained intact and was even slightly expanded at the anteriormost part of the forelimb bud (Fig. 2B). This expansion is unlikely to be solely related to the diminution of Msx activity in the AER, since it was not observed in the AER of the compound $M s x 1^{\text {null/null }} M s x 2^{+/ n u l l}$ mutants (Fig. 2D), but suggests a role of Msx in the mesenchyme for AER formation. 


\section{The Prx1-Cre Msx $1^{\text {null/null }} M s x 2^{\text {null/Flox }}$ mutants display abnormal digit formation and preaxial polydactyly}

Contrary to the double Msx $1^{\text {null/null }} M s x 2^{\text {null/null }}$ mutants, the Msx conditional double mutants were viable until birth, allowing skeleton preparations to be performed at $18.5 \mathrm{dpc}$ (Fig. 3). At this stage, the three limb segments, i.e. the stylopod (arm or thigh), zeugopod (forearm or leg) and autopod (hand or foot), were present in both fore- and hindlimbs (Fig. 3A-D), but several abnormalities were noticeable along both the proximodistal (PD) and anteroposterior (AP) axes. Along the PD axis, abnormalities were similar in fore- and hindlimbs. They were limited to the autopod where the distal phalanges (the last one and occasionally the penultimate) were absent or incompletely developed, and devoid of calcification (Fig. 3E-H). This was confirmed by the absence of nails (normally borne by the last phalanx) in the mutants (Fig. 3I-L). This reduction of digit extremities was similarly observed in the double Msx $1^{\text {null/null }} M s x 2^{\text {null/null }}$ mutant (Lallemand et al., 2005).

Along the AP axis, the phenotype was much more striking in fore- than in hindlimbs. In the forelimbs, the AP defects involved the three segments (Fig. 3A,C). In the stylopod, we observed a systematic absence of the deltoid tuberosity. In the zeugopod, the anterior bone (radius) was absent in half of the specimens analyzed. In the other half, radius phenotypes ranged from a reduction in width to shortening and bending or truncation (Fig. 3C and Supplementary Fig. S2A). In the autopod, preaxial polydactyly was systematic, with up to 8 digits or digit-like elements (Fig. 3F and Supplementary Fig. S2B). Based on their relationships to the underlying carpals, the four posteriormost digits could be considered as digits 2 to 5 . Anterior to digit 2, we systematically observed a cartilaginous structure that we interpret as the non-ossified metacarpal of the misdeveloped thumb. This structure was unique in half of the cases and duplicated in the other half. Finally, the anteriormost digit displayed a 
morphology similar to digit 2,3 or 4 . In a minority of cases (16\%) two such digits were observed (Fig. 3F and Supplementary Fig. S2B).

The phenotype of the hindlimbs was more discrete. The stylopod was always normal, as was the zeugopod in two thirds of the specimens analyzed (Fig. 3D and Supplementary Fig. S2C). In the remaining third, the tibia was shortened or, in a few cases, absent (Supplementary Fig. S2C). The AP abnormalities of the hindlimb autopod were also very mild. Because of the reduced development of the terminal phalanges in all digits, no three-phalanged big toes (halluces) could be identified, in contrast to what is seen in the Msx1 $1^{\text {null/null }} M s \times 2^{+/ n u l l}$ compound mutants (Lallemand et al., 2009). In addition, in more than 50\% of the cases, the autopod was strictly pentadactylous (Supplementary Fig. S2D). In the other specimens analyzed, the only abnormality observed along the AP axis was the anterior presence of a small cartilaginous formation, either independent of or branched on digit 1 (Fig. $3 \mathrm{H}$ and Supplementary Fig. S2D). Considering this, and the fact that the three-phalanged toe of the $M s x 1^{\text {null/null }} M s \times 2^{+/ n u l l}$ compound mutants can be considered as the mildest manifestation of an anterior polydactyly, the hindlimb phenotype of the conditional double mutants can be viewed as a slight aggravation of the hindlimb phenotype of the $M s x 1^{\text {null/null }} M s \times 2^{+/ n u l l}$ compound mutants.

The differences in skeletal malformations seen in the double conditional mutant fore- and hindlimbs may result in part from the properties of the Prxl-Cre transgene. This transgene is known to be expressed later and to a lesser extent in the hindlimb than in the forelimb (Logan et al., 2002; see also Discussion). Due to these technical limitations, and since our conditional knockout strategy aimed primarily to decipher the role of Msx genes in the anterior region of the limb, we focused the rest of our study on the analysis of the AP phenotype in the forelimb. 


\section{Alteration of the anteroposterior polarity in the forelimbs of the Msx conditional double mutants}

The phenotype observed in the autopod of the conditional double mutant forelimb can be viewed as a partial mirror-image duplication of the digits. This was confirmed by analysis of several genetic markers expressed in a polarized pattern along the AP axis. At $11.5 \mathrm{dpc}$ and $12.5 \mathrm{dpc}$, Hoxd12 is normally expressed exclusively in a posterior domain of the autopod, corresponding to presumptive digits 2 to 5 (Fig. 4A,C). In the conditional mutant forelimb, this posterior domain was not modified but Hoxd12 was further expressed in an ectopic domain at the anterior margin of the deformed autopod (Fig. 4B,D). The two domains were separated by a Hoxd12 non-expressing one. The ectopic anterior expression domain is likely to correspond to the anteriormost extra-digits with a posterior morphology, whereas the negative region would match with the presumptive territory of the thumb-like structures observed in the mutant new-borns (compare Fig. 4B and Fig. 3F). Similar results were observed for Hoxd11 (Fig. 4E,F) as well as for Hand2 (Fig. 4G,H), another posterior-specific marker. It should be noted that such anterior ectopic expression domains were not observed in the hindlimbs of conditional mutants where all these genes showed only an anterior extension in expression, similar to what was observed in the $M s x 1^{\text {null/null }} M s \times 2^{+/ n u l l}$ compound mutant (data not shown).

In wild-type embryos, $\operatorname{Pax} 9$ is expressed at $11.5 \mathrm{dpc}$ in a domain corresponding to the presumptive digit 1 (Neubuser et al., 1995). In the forelimb of the conditional double mutant, Pax9 was dramatically downregulated but remained expressed at a low level in a small, proximal region (Fig. 4I,J). This region corresponds to the Hoxd12 negative domain that likely gives rise to thumb-like structures in the newborn autopod.

Altogether, gene expression results confirm that the Msx conditional double mutants display a partial anterior mirror-image duplication of the forelimb digit territory. The anteriormost 
digits display posterior features whereas the intermediate structures, generally limited to metacarpals, are likely to be thumb-like formations.

In the Msx conditional double mutant, $S h h$ is ectopically expressed in the anteriormost region of the limb bud mesenchyme, in spite of Etv4/Etv 5 upregulation

In the Msx conditional double mutant, gene expression abnormalities as well as anterior polydactyly are reminiscent of phenotypes due to Sonic hedgehog (Shh) ectopic expression anteriorly (Sharpe et al., 1999; Lettice et al., 2003; Sagai et al., 2004; Mao et al., 2009; Zhang et al., 2009). In normal embryos, Shh expression is restricted to a small region of the posterior mesenchyme corresponding to the zone of polarizing activity (ZPA) (Fig. 5A). At 11.5 dpc, we observed a Shh ectopic expression at the anterior of the forelimb buds of mutant embryos (5/5), albeit at a low level (Fig. 5B). In addition, ectopic activation of Gli1, a direct target of Shh, was detected at the anterior margin of the bud mesenchyme, confirming functional Shh signaling (Fig. 5C,D).

Etv4/Etv5 double and Msx conditional double mutants share a strikingly similar limb phenotype, with anterior polydactyly and formation of an ectopic expression domain for posterior markers such as Hand2 and Gli1. It has been demonstrated that this phenotype is due to ectopic activation of Shh anteriorly (Mao et al., 2009; Zhang et al., 2009). We therefore investigated a possible downregulation of the Etvs in the Msx conditional double mutant that might explain its phenotype. ISH actually demonstrated the reverse. Etv4 and Etv5 expression was not modified at $10.75 \mathrm{dpc}$ (Supplementary Fig. S3) and, at $11.5 \mathrm{dpc}$, the two genes were clearly upregulated anteriorly (Fig. 5E-H) where Shh ectopic expression takes place. Both Etv4 and Etv5 are positively controlled by FGF signaling, and their anterior upregulation is consistent with the anterior expansion of the Fgf8 expression domains in the Msx double 
conditional mutants (Fig 2B). But even at high expression levels, Etv4 and Etv5 cannot repress ectopic expression of Shh in the Msx mutant context (see Discussion).

\section{Global signaling activity modification in the anteriormost region of the limb bud mesenchyme of the Msx conditional double mutant}

The Msx conditional double mutant phenotype displays features different from those associated with Shh misexpression, such as observed in the Hemimelic extra-toes $\left(S h h^{H x}\right)$ mutant. In the latter, a point mutation in the ZPA regulatory sequence (ZRS), a long-range regulatory sequence of $S h h$ (Lettice et al., 2003; Sagai et al., 2004) leads to to the formation of an ectopic anterior Shh expression domain, resulting in a mirror-image duplication of the digits in all four limbs, and a reduction or agenesis of the anterior bone of the zeugopod (radius, tibia). The latter is more severe in the hindlimbs than in the forelimbs (Knudsen and Kochhar, 1981), whereas the reverse situation is observed in the Msx conditional double mutant. In addition to these phenotypic differences, defects in AP polarity do not seem to be brought about the same way. At $10.75 \mathrm{dpc}$ (40-42 pairs of somites), Shh expression could not be detected by ISH in the anterior region of the limb mesenchyme in either of the two mutants (Fig. 6A-C). Similarly Gli1, Hand2 or Hoxd12 were expressed only in their normal posterior domain (Fig. 6D,E and data not shown). Nevertheless, ectopic Fgf4 expression in the AER indirectly demonstrated anterior polarizing activity in the Msx conditional double mutants. At $10.75 \mathrm{dpc}$, in normal embryos, $\mathrm{Fgf4}$ is expressed in the posterior aspect of the AER, but not in its anterior third (Fig. 6A,D), as its expression depends on Shh signaling from the posterior mesenchyme. At this stage, Fgf4 expression appeared normal in $S h h^{H x /+}$ mutant forelimbs (Fig. 6C) whereas conspicuous, ectopic expression was observed at the anterior side of the AER in the Msx conditional double mutant (Fig. 6B,E). This difference could not be explained by minor variations in embryo development, as ectopic Fgf4 expression was also 
detected in slightly delayed Msx mutant embryos (compare Fig. 6B and 6C). Likewise, it was not solely due to the reduced Msx activity in the AER of the Msx conditional double mutant since Fgf4 expression in the $M s x 1^{\text {null/null }} M s x 2^{+/ n u l l}$ compound mutant forelimb showed no difference as compared to control at $10.75 \mathrm{dpc}$ (Fig. 6F).

It should be considered that ISH is a poorly quantitative technique. Thus, at $10.75 \mathrm{dpc}$, although not revealed by ISH, Shh mRNA might already accumulate anteriorly, and at a higher level in the Msx double mutants than in the $S h h^{H x /+}$ mutants. This would induce Fgf4 expression earlier in the former than in the latter. To assess this point, we dissected the anterior part of limb buds from $S h h^{H x /+}$ and Msx conditional double mutant embryos at 10.75 dpc, and analyzed the Shh expression by quantitative RT-PCR. In both mutants, the level of Shh RNA in the anterior limb mesenchyme was abnormally high compared to their wild-type littermates (10 to 15 times higher) (Supplementary Fig. S4). Using the limb posterior region of wild-type embryos as a reference, we estimated the anterior/posterior Shh RNA level ratio to be around $0.1 \%$ in normal embryos (negative controls) whereas it was around 1 to $2 \%$ in the $S h h^{H x /+}$ or the Msx conditional double mutants (data not shown). Nevertheless, this level was not significantly different between $S h h^{H x /+}$ and Msx conditional double mutants at this stage (Supplementary Fig. S4), thus excluding earlier activation of $F g f 4$ by Shh in the latter. BMP signaling has long been recognized to negatively regulate Fgf4 expression in the AER (Pizette and Niswander, 1999; Zuniga et al., 1999; reviewed in Dahn and Fallon, 1999), and Msx genes are involved in BMP signaling at several sites during development and may even regulate Bmp4 expression (Chen et al., 1996; Marazzi et al., 1997; Bei and Maas, 1998; Zhang et al., 2002; Ogawa et al., 2006). We thus analysed the three Bmp genes expressed in limb development (Bmp2, Bmp4, Bmp7) as well as the BMP antagonist gene Gremlin, which has been shown to play a crucial role during limb development (Khokha et al., 2003). At 11.5 dpc expression of these four genes was modified at the anterior of the forelimb bud, but in 
different ways: Bmp4 was downregulated, whereas $B m p 7$ was moderately upregulated and Bmp2 and Gremlin ectopically expressed (Fig. 7). In normal embryos, at $11.5 \mathrm{dpc}$, Bmp4 is expressed in the mesenchyme underlying the AER, with a stronger level of expression posteriorly (Fig. 7A). In the Msx conditional double mutant, only the posterior domain of expression remained whereas the anterior region of the limb mesenchyme was almost entirely devoid of Bmp4 transcripts (Fig. 7B). This downregulation cannot be attributed to the anterior ectopic expression of Shh since Bmp4 is not downregulated in the $S h h^{H x /+}$ mutant at the same stage (Fig. 7C). Bmp2 (Fig. 7D), and Gremlin (Fig. 7J) are normally not expressed in the anteriormost part of the limb bud at $11.5 \mathrm{dpc}$. In the Msx mutant, a tiny spot of $B m p 2$ expression was visible at the anterior tip of the AER (Fig. 7E) whereas, for Gremlin, the anterior ectopic expression was larger and located in the mesenchyme (Fig. 7K). For Bmp7, it is normally expressed in the whole limb bud mesenchyme at $11.5 \mathrm{dpc}$, but more intensively in the posteriormost region than in the anteriormost one (Fig. 7G). In the Msx conditional double mutant, $B m p 7$ expression was slightly upregulated anteriorly so that the level of staining was similar at the anterior and posterior aspects of the limb bud (Fig. $7 \mathrm{H})$. For all three genes (Bmp2, Bmp7 and Gremlin) the modification was similar in the Msx conditional double mutant and the $S h h^{H x /+}$ mutant (compare Fig. $7 \mathrm{E}$ and $7 \mathrm{~F}, 7 \mathrm{H}$ and $7 \mathrm{I}, 7 \mathrm{~K}$ and $7 \mathrm{~L}$ ). Contrary to what was observed for Bmp4, the modifications in expression of these genes seem to be a consequence of the Shh anterior ectopic expression.

At 10.75 dpc, the situation was different. Bmp2 and Bmp4 were similarly expressed in control and Msx mutant embryos (Supplementary Fig. S3E-H). Bmp7 was slightly upregulated anteriorly compared to control embryos, but this difference was also observed in stagematched $S h h^{H x /+}$ mutants (Fig. 7M-O). For Gremlin, a clear anterior ectopic expression domain was observed but, as for $B m p 7$, a similar result was obtained with the $S h h^{H x /+}$ mutant (Fig. 7P-R). Thus, at $10.75 \mathrm{dpc}, B m p 7$ anterior upregulation, and Gremlin ectopic expression, 
must be due, in both kinds of mutants, to the low level of Shh ectopically present in the anteriormost region of the limb bud mesenchyme.

In conclusion, with regard to the BMP signaling pathway, the only noticeable difference between Msx conditional double mutant and $S h h^{H x /+}$ mutant embryos is the anterior downregulation of Bmp4 expression at $11.5 \mathrm{dpc}$ (see Discussion). This can explain the difference of phenotype between the two types of mutants but not the anterior ectopic expression of $\mathrm{Fgf4}$, which is visible, at $10.75 \mathrm{dpc}$, only in the AER of the Msx conditional double mutant (Fig. 6B,E).

\section{Apoptosis and cell proliferation}

To assess the respective role of apoptosis and cell proliferation in the anterior overgrowth of the mutant limb bud, we analysed them by immunofluorescence on limb bud sections at 11.5 dpc (Fig. 8). Analysis of active Caspase-3 revealed that the region of intense apoptotic activity, that is normally observed at the anterior aspect of the limb bud of wild-type embryos (Fernández-Terán et al., 2006) (Fig. 8A), was lost in the Msx conditional double mutant (Fig. 8B). Conversely, the central domain of apoptosis was present in both kind of embryos. This is similar to what was observed in the hindlimbs of the $M s x 1^{\text {null/null }} M s \times 2^{+/ n u l l}$ compound mutant (Lallemand et al., 2009).

Analysis of cell proliferation on the same specimens, using an anti-phospho-histone $\mathrm{H} 3$ antibody, did not reveal differences between control (Fig. 8C) and Msx conditional double mutant embryos (Fig. 8D). Nevertheless, during this period of development, cell proliferation is so intense in the limb mesenchyme that small differences in the growth rate are difficult to assess (see also Discussion). 


\section{DISCUSSION}

In this report, we describe the consequences of mesenchyme-specific abrogation of all Msx activity in the limb bud. Our analysis is focused on the AP development of the mutant limb and more specifically of the most anterior digit (digit 1) territory, because this region is profoundly altered in the double Msxl Msx2 null mutant. Our results show that Msx activity in the limb bud mesenchyme is critical for the proper development of the anteriormost region and reveal new roles for Msx genes in two major signaling pathways, namely Shh and BMP.

\section{AER development is complete in the Prx1-Cre Msx1 ${ }^{\text {null/null }}$ Msx $2^{\text {null/Flox }}$ conditional mutant}

The territory corresponding to the most anterior digit is lost early in the double Msx $1 M s x 2$ null mutant, following failure of the AER to form anteriorly (Lallemand et al., 2005). In contrast, the AER develops along its entire length in the Msx conditional double mutant and, consequently, the anteriormost region of the mesenchyme does not regress. This may indicate that Msx genes act cell-autonomously in the ectoderm at early stages of limb development to promote AER formation anteriorly. However, alternative hypothesis may be considered, especially in the light of data showing that abrogating BMP signaling in the AER abolishes Msx2 AER-specific expression without altering AER integrity (Wang et al., 2004; Maatouk et al., 2009). Although Msxl expression was not investigated in these studies, this may suggest that Msx genes are dispensable in the ectoderm for formation of the AER. AER forms at the boundary between dorsal and ventral domains in the ectoderm. Initially, DV polarity is established in the mesoderm, then transferred to the ectoderm (Altabef et al., 1997; Michaud et al., 1997). This transfer likely involves the BMP pathway (Ahn et al., 2001; for a discussion, see Robert, 2007). It should be noted that the Prx1-Cre transgene activity takes place progressively in both fore- and hindlimbs (Logan et al., 2002), and that, at the earliest 
stages of limb outgrowth, some Msx activity is likely to remain in the mesenchyme of our conditional mutant. A recent paper (Zhang et al., 2010) has shown that for Twistl, a gene specifically expressed in the limb bud mesoderm, the Prxl-Cre-induced null mutation leads to a less dramatic phenotype than the constitutive null mutation. In particular, AER development is complete in the conditional null mutant and impaired in the constitutive one. This is likely due to residual Twist 1 activity at $9.5 \mathrm{dpc}$ in the mesoderm. We therefore cannot exclude that the anterior truncation of the AER observed in the Msxl Msx2 double null mutant is due to a mesenchyme-bound, non-cell autonomous role for Msx in DV information transfer, in keeping with frequent Msx involvement in the BMP pathway. In the Msx conditional double mutant, Prxl-Cre expression would take place too late in the mesoderm to interfere with this process.

\section{Phenotypic alterations in the mesenchyme-specific Msx mutant}

Skeleton analysis of the conditional mutants clearly revealed different phenotypes between fore- and hindlimbs along the AP axis but a similar one along the PD axis. This can be explained by the expression properties of the Prxl-Cre transgene, which is active earlier in fore- than hindlimbs (Logan et al., 2002). This difference is particularly obvious before 10.5 dpc. Later on, the recombination of floxed alleles appears almost complete in both fore- and hindlimbs. Complete inactivation of the Msx2 floxed allele might therefore occur too late (after $10.5 \mathrm{dpc}$ ) in the hindlimb to drastically alter its development along the AP axis. Thus the AP abnormalities observed in the hindlimbs may be due primarily to the reduction of Msx activity in limb bud ectoderm and mesoderm, as in the Msx $1^{\text {null/null }} M s x 2^{+/ n u l l}$ compound mutant, slightly enhanced by the progressive loss of all Msx activity in the mesenchyme. The loss of Msx activity is likely to be more complete in the forelimb because of the earlier and more extensive activity of the Prxl-Cre transgene. 
Conversely, the formation of the last phalanges is a late event in limb morphogenesis (SanzEzquerro and Tickle, 2003). At this stage, the Cre-induced inactivation of the Msx2 conditional allele is likely to be complete in both fore- and hindlimbs, leading to similar phenotypes in both. Nevertheless, several mutations have been reported that lead to different AP phenotypes in fore- and hindlimbs (e.g. $S h h^{H x /+}$ : Knudsen and Kochhar, 1981; Blanc et

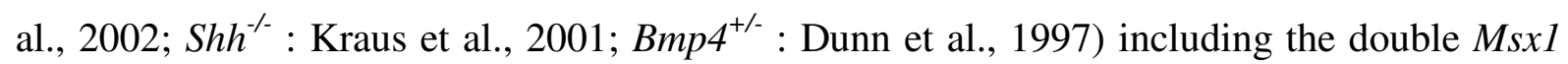
Msx2 null mutation (Lallemand et al., 2005). It thus remains possible that the phenotypic variations observed between fore- and hindlimb buds are due to slightly different roles for Msx genes in either of these.

The most dramatic outcome of the mesenchyme-specific Msx mutation is an anterior outgrowth of the mesoderm that results in anterior polydactyly. This raises the question of which changes in local cell physiology are underlying this morphological alteration. We observed that the apoptotic domain that normally forms anteriorly (Fernández-Terán et al., 2006) is missing in our mutant. This is in keeping with similar absence of this domain that we observed in the hindlimb of a hypomorphic Msx $1^{\text {null/null }} M s \times 2^{+/ \text {null }}$ mutant (Lallemand et al., 2009). Conversely, we did not observe changes in cell proliferation that similarly might underlie outgrowth. However, contrary to apoptosis, which is restricted in time and space in the developing limb bud, proliferation is active in the whole limb bud at the stage of development we analysed and lasts over several days, such that subtle changes, not detectable in our analysis, might be cumulative. It is highly plausible that proliferation plays a role in anterior polydactyly, but, to establish it, it would be necessary to conduct a systematic analysis of cell proliferation in 3D at different stages of development, the kind of which is just starting to be accessible (Boehm et al., 2010; Gros et al., 2010; Wyngaarden et al., 2010).

\section{Control of Shh expression}


The most striking result of this study is the demonstration of a role for Msx genes in the control of Shh repression anteriorly. This was suggested by our previous work on the $M s x 1^{\text {null/null }} M s x 2^{\text {null/null }}$ double mutants, but Shh ectopic expression in these mutants was inconsistent (Lallemand et al., 2005). On the contrary, we could detect ectopic Shh expression in the forelimbs of all Msx conditional double mutant embryos we analyzed at $11.5 \mathrm{dpc}(5 / 5)$. This abnormal expression of Shh was confirmed by quantitative RT-PCR at $10.75 \mathrm{dpc}$. It has been shown that, in the limb, positive and negative control of Shh transcription depends on a long-range regulatory sequence referred to as the ZPA regulatory sequence (ZRS) (Lettice et al., 2003; Sagai et al., 2004). Preliminary experiments to investigate potential binding of Msx proteins to the ZRS, that would suggest direct control of Shh expression, did not give conclusive results (data not shown). This does not exclude potential Msx interaction with the ZRS, considering that this interaction may involve several proteins engaged in a complex. In this respect, it should be noted that Gli3R, the truncated form of the Gli3 protein, accumulates in the anteriormost region of the limb, where Msx activity is high, and that $\mathrm{Gli3}^{-/}$mutant embryos also display ectopic Shh expression anteriorly in the limbs (Buscher et al., 1997). Furthermore, Gli3R has been shown to interfere with positive transcriptional regulation at the ZRS (Galli et al., 2010). Interactions between Gli3R and Msx might be required for Shh repression anteriorly. Alternatively, Shh may be activated by ectopic expression of Hand 2 in the Msx double conditional mutant. To date, we could not establish a temporal hierarchy in the activation of Hand2 vs. Shh.

Etv4 and Etv5 are two transcription factors that also repress Shh anteriorly in the limb, and the phenotype of Etv4/Etv5 double null mutants strikingly resemble this of Msx conditional double mutants (Mao et al., 2009; Zhang et al., 2009), which might suggest epistatic relations between Etv and Msx. This however is unlikely, as Msx1 remains expressed in Etv4 Etv5 double null mutant limb buds (Xin Sun, personal communication). Conversely, Etv4 and Etv5 
are not downregulated in our Msx conditional mutant. On the contrary, their expression is upregulated and expanded in the anterior limb mesenchyme, in accordance with the increase in FGF signaling from the AER. It should be noticed that, in the Twist1 null mutant, Etv4 and Etv 5 are also upregulated in the anterior limb bud mesenchyme, which does not preclude Shh derepression anteriorly. Indeed, it has been recently demonstrated that Etv and Twist1 proteins form a complex that is required to repress Shh anteriorly (Zhang et al., 2010). The similarity between these and our results may suggest a similar mechanism for anterior repression of Shh by Msx.

\section{BMP signaling and control of Bmp4 expression,}

A number of studies have focused on the relationships between BMP signaling and the Msx gene family. These relationships appear to be complex and context-dependent, with BMP or Msx acting either upstream or downstream of each other depending on the organ and/or the process analyzed (Chen et al., 1996; Marazzi et al., 1997; Bei and Maas, 1998; Zhang et al., 2002). In the limb, most of the results published describe Msxl and Msx2 as BMP signaling targets. Concerning Msx2, inactivation of a Bmp4 conditional mutant allele in the limb mesenchyme, using Prxl-Cre, abolishes its expression in the overlying ventral limb ectoderm (Selever et al., 2004). A similar result was observed by generating an ectoderm-specific knockout of the Bmprla that prevents ectodermal cells to respond to BMP signaling (Ahn et al., 2001). Reciprocally, AER-specific null mutation of Bmp2 and Bmp4 provokes Msx2 downregulation in the underlying mesenchyme (Maatouk et al., 2009). Furthermore, a BMPresponsive enhancer, which is sufficient for Msx2-like expression of a reporter in the limb bud, has been identified at the 5' end of the Msx2 first exon (Brugger et al., 2004).

Conversely, both $M s x 1$ and $M s x 2$ are upregulated in the limb mesenchyme by a null mutation of Gremlin, a potent BMP inhibitor expressed in the limb (Khokha et al., 2003; Michos et al., 
2004). Nevertheless, although a mesenchyme-specific null mutation of Bmprla leads to a drastic downregulation of the two Msx genes (Ovchinnikov et al., 2006), residual expression is still detectable for both $M s x 1$ and $M s x 2$ anteriorly, raising the possibility that, in this region, Msx genes are controlled by other factors such as, for example, Gli3R (Lallemand et al., 2009). Indeed, it has been shown that the Talpid ${ }^{2}$ chick mutation, which precludes proper processing of Gli3 into Gli3R (Wang et al., 2000) also abolishes $M s x 2$ expression and leads to non polarized Msxl expression in the mesoderm (Krabbenhoft and Fallon, 1992). Most of the results quoted here, however, suggest that Msx might work as downstream effectors in the BMP pathway for most of the limb mesenchyme, and indeed, the Fgf4 expression domain in the AER extends anteriorly in a similar way in the Msx conditional double mutant and the Bmp4 conditional null mutant (Selever et al., 2004; this work). This suggests that BMP signaling is impaired in the Msx conditional double mutant.

However, the phenotype of the Msx conditional double mutant is quite different from those of the mesenchyme-specific Bmp4 and Bmprla null mutants (Selever et al., 2004; Ovchinnikov et al., 2006). In either of these mutants, the hindlimbs are more affected than the forelimbs, when the mutation is induced using the Prxl-Cre transgene. Bmp4 mutants display pre- and postaxial polydactyly and no alteration of the zeugopod. In the Bmprla mutant, there is a severe dysplasia of the autopod in fore- and hindlimb and, furthermore, frequent disappearance of the fibula in the hindlimb. In addition, in these two mutants, patterning along the AP axis is not affected to such an extent as in the Msx conditional double mutant. Hoxd11, Hoxd12 or Hoxd13 expression domains extend only slightly anteriorly, and no ectopic Shh expression is detected. Therefore, in the limb mesenchyme, Msx genes cannot be considered exclusively as downstream effectors in the BMP signaling pathway.

The possible control of Bmp genes by Msx activity in the limb is less documented. It has been shown that forced expression of Msx2 in the mesoderm of chick limb bud induces Bmp4 
misexpression (Ferrari et al., 1998). The results described in this report show that, in the anterior region, Msx activity is not required for initial Bmp4 expression, but rather for its maintenance from $11.5 \mathrm{dpc}$ on. This corroborates previous studies showing that Msx1 interacts with Pax9 to synergistically transactivate, in vitro, a Bmp4 promoter sequence (Ogawa et al., 2006). Indeed, genetic interactions between MsxI and Pax9 were recently demonstrated to regulate morphogenesis of the tooth, where the two genes are expressed (Nakatomi et al., 2010). Furthermore, in this organ, defects induced by Msx1 Pax9 double heterozygous null mutations could be partially rescued by a Bmp4 transgene. Pax $9, M s x 1$ and $M s x 2$ are also coexpressed in the presumptive territory of the thumb at $11.5 \mathrm{dpc}$ (Neubuser et al., 1995), and a Pax9 null mutation, similarly to Msx gene conditional mutation, leads to preaxial digit duplication (Peters et al., 1998). Our results confirm that, in the limb, Msx activity controls Pax9 expression, as we previously showed (Lallemand et al., 2009), and second, suggest that in this field too, Msx and Pax9 proteins might interact to control Bmp4 expression.

Contrary to Bmp4, Bmp2 and Bmp7, the other two Bmp genes expressed in the limb bud, were upregulated anteriorly in the Msx conditional double mutant. However, in both cases, these modifications were very modest and, considering the concomitant anterior ectopic expression of the BMP-antagonist Gremlin, it is likely that, in the anteriormost limb region, BMP signaling is globally diminished in our Msx mutant. Moreover, all the expression differences observed, except the Bmp4 downregulation, are similar in the Msx conditional double mutant and the $S h h^{H x /+}$ mutant embryos at both 10.75 and $11.5 \mathrm{dpc}$. Thus the anterior downregulation of Bmp4 expression at $11.5 \mathrm{dpc}$ is the only process that can account for the difference of phenotype between the two kinds of mutants. Nevertheless, it does not explain the first manifestations of the abnormal phenotype in the Msx conditional double mutant, namely the 
anterior ectopic expression of Fgf4 in the AER, which could be detected as early as 10.75 dpc.

\section{Conclusion}

The AP limb phenotype of the Prx1-Cre Msx $1^{\text {null/null }} M s x 2^{\text {null/Flox }}$ mutants can be explained in part by the anterior de-repression of $S h h$, but also by the downregulation, in the same region, of Bmp4. The fact that Msx proteins can play a role in two different signaling pathways (Shh and BMP) in two opposite ways (repression or activation) suggests they cooperate with different protein partners. Pax9 is a likely candidate for the control of Bmp4 expression. Concerning Shh repression, Gli3R and Etv are possible candidates. Further investigation at the protein level will be necessary to clarify this issue.

\section{EXPERIMENTAL PROCEDURES}

\section{Mice and embryos}

Generation of the Msxl mutant and the Msx2 conditional mutant alleles has been described previously (Houzelstein et al., 1997; Bensoussan et al., 2008). The Prxl-Cre transgene (a kind gift of Malcolm Logan) was introduced to the $M s x 1^{+/ n u l l} M s x 2^{+/ n u l l}$ background. Subsequently, the combined Prxl-Cre Msxi ${ }^{+/ n u l l} M s x 2^{+/ n u l l}$ strain was maintained on an outbred (NMRI) background. Conditional mutants were obtained by crossing Prxl-Cre Msx ${ }^{+/ n u l l} M s x 2^{+/ n u l l}$ males with $M s x 1^{+/ n u l l} M s x 2^{\text {Flox/Flox }}$ females. Control embryos was chosen among the littermates of the conditional mutants possessing at least one wild-type allele of each Msx gene (PrxlCre Msx ${ }^{+/+} M s x 2^{+/ F l o x}$ or Prxl-Cre Msx $1^{+/ n u l l} M s x 2^{+/ F l o x}$ genotypes). The Prxl-Cre transgene is carried by the male because of its expression in the female germline (Logan et al., 2002). Day of the plug was considered as $0.5 \mathrm{dpc}$. 


\section{Whole-mount in situ hybridization}

Whole-mount in situ hybridization (ISH) was performed as described previously (Houzelstein et al., 1997). RNA probes were generated by transcription of DNA templates using Sp6, T3 or T7 RNA polymerase. The following DNA templates were prepared by cDNA PCR amplification followed by cloning into the pGEM $^{\circledR}-\mathrm{T}$ vector (Promega) : Fgf8 (complete cDNA sequence), Msx2 (complete exon 2 sequence) and Gli1 (complete exon 13 sequence). Bmp2, Bmp4 and Bmp7 were a gift from B. Hogan, Etv4 and Etv5 from X. Sun, Fgf4 from G. Martin, Gremlin from R. Zeller, Hoxd11 and Hoxd12 from P. Dollé, Pax9 from R. Balling, Ptcl and Shh from A. McMahon, Gli3 from U. Rüther, Dlx5 from D. Acampora and Hand2 from E. Olson.

\section{Skeletal preparation}

After euthanasia, newborns were eviscerated and their bodies placed in water at $70^{\circ} \mathrm{C}$ for 50 min to permit complete removal of the skin. Embryos were stained overnight in Alizarin red/Alcian blue solution, then fixed $1 \mathrm{hr}$ in $95 \%$ ethanol, cleared in $1 \% \mathrm{NaOH}$, and stored in PBS at $4^{\circ} \mathrm{C}$.

\section{Cell death and cell proliferation analysis}

Cell death and cell proliferation were analysed on frozen histological sections (14 $\mu \mathrm{m}) \mathrm{using}$, respectively, an anti-active Caspase-3 (BD Pharmigen) and an anti-phospho-histone H3 (Cell Signaling) according to the manufacturer's protocol.

\section{Quantitative Real-time PCR (qRT-PCR)}


Anterior and posterior fragments of limb buds were dissected separately from $10.75 \mathrm{dpc}$ embryos. For Msxl Msx2 conditional double mutants, 7 mutant and 7 control littermates were used ; for $H x$ mutants, 7 mutant and 5 control littermates were used. Total RNA was isolated using an RNeasy Mini Kit (Qiagen) following the manufacturer's instructions. cDNA were synthesized from 100ng of RNA using the SuperScript III reverse transcriptase (Invitrogen) and real-time PCR was carried out using the SYBRGreen PCR master mix (Applied Biosystems) and an Applied Biosytems Step One plus cycler, according to the manufacturer's instructions. PCR cycle parameters were: 10 minutes at $95^{\circ} \mathrm{C}$ (initial incubation), followed by 15 seconds at $95^{\circ} \mathrm{C}, 1$ minute at $60^{\circ} \mathrm{C}$ for 40 cycles. Posterior fragments from wild-type embryos were used as positive controls to set up the baseline for normal Shh expression in our qRT-PCR conditions. The Gapdh gene was used to normalize results. Primer sequences were : qSHH-for : TGCTGGCTCGCCTGGCTG ; qSHH-rev : AAACAGCCGCCGGATTTGGC ; qGAPDH-for : $\quad$ GGCAAAGTGAGATTGTTC ; $\quad$ qGAPDH-rev : AATTTGCCGTGAGTGGAGTC. PCR efficiency was in the range of $98 \%$ to $100 \%$ for all assays.

\section{ACKNOWLEDGMENTS}

We are very grateful to Dr. X. Sun for fruitful discussions and sharing of unpublished results, and to Dr C. Christ and M. Lopes for critical reading of the manuscript. This work was supported by the Institut Pasteur, the Centre National de la Recherche Scientifique, and by grant ANR-06-MRAR-027-02 from the Agence Nationale de la Recherche. V. B-T. was the recipient of a fellowship from the Institut Weizmann des Sciences France-Europe.

\section{REFERENCES}


Ahn K, Mishina Y, Hanks MC, Behringer RR, Crenshaw EB, 3rd. 2001. BMPR-IA signaling is required for the formation of the apical ectodermal ridge and dorsal-ventral patterning of the limb. Development 128:4449-4461.

Altabef M, Clarke JD, Tickle C. 1997. Dorso-ventral ectodermal compartments and origin of apical ectodermal ridge in developing chick limb. Development 124:4547-4556.

Bei M, Maas R. 1998. FGFs and BMP4 induce both Msx1-independent and Msx1-dependent signaling pathways in early tooth development. Development 125:4325-4333.

Bendall AJ, Abate-Shen C. 2000. Roles for Msx and Dlx homeoproteins in vertebrate development. Gene 247:17-31.

Bensoussan V, Lallemand Y, Moreau J, Cloment CS, Langa F, Robert B. 2008. Generation of an Msx2-GFP conditional null allele. Genesis 46:276-282.

Blanc I, Bach A, Robert B. 2002. Unusual pattern of Sonic hedgehog expression in the polydactylous mouse mutant Hemimelic extra-toes. Int. J. Dev. Biol. 46:969-974.

Boehm B, Westerberg H, Lesnicar-Pucko G, Raja S, Rautschka M, Cotterell J, Swoger J, Sharpe J. 2010. The role of spatially controlled cell proliferation in limb bud morphogenesis. PLoS Biol 8:e1000420.

Brugger SM, Merrill AE, Torres-Vazquez J, Wu N, Ting MC, Cho JY, Dobias SL, Yi SE, Lyons K, Bell JR, Arora K, Warrior R, Maxson R. 2004. A phylogenetically conserved cis-regulatory module in the Msx2 promoter is sufficient for BMPdependent transcription in murine and Drosophila embryos. Development 131:51535165.

Buscher D, Bosse B, Heymer J, Ruther U. 1997. Evidence for genetic control of Sonic hedgehog by Gli3 in mouse limb development. Mech Dev 62:175-182.

Chen Y, Bei M, Woo I, Satokata I, Maas R. 1996. Msx1 controls inductive signaling in mammalian tooth morphogenesis. Development 122:3035-3044.

Crossley PH, Martin GR. 1995. The mouse Fgf8 gene encodes a family of polypeptides and is expressed in regions that direct outgrowth and patterning in the developing embryo. Development 121:439-451.

Dahn RD, Fallon JF. 1999. Limbiting outgrowth: BMPs as negative regulators in limb development. Bioessays 21:721-725.

Davidson D. 1995. The function and evolution of Msx genes: pointers and paradoxes. Trends Genet 11:405-411.

Dunn NR, Winnier GE, Hargett LK, Schrick JJ, Fogo AB, Hogan BL. 1997. Haploinsufficient phenotypes in Bmp4 heterozygous null mice and modification by mutations in Gli3 and Alx4. Dev Biol 188:235-247.

Fernández-Terán MA, Hinchliffe JR, Ros MA. 2006. Birth and death of cells in limb development: a mapping study. Dev. Dyn. 235:2521-2537.

Ferrari D, LIchtler AC, Pan ZZ, Dealy CN, Upholt WB, Kosher RA. 1998. Ectopic expression of Msx2 in posterior limb bud mesoderm impairs limb morphognesis while inducing Bmp4 expression, inhibiting cell proliferation, and promoting apoptosis. Dev. Biol. 197:12-24.

Galli A, Robay D, Osterwalder M, Bao X, Benazet JD, Tariq M, Paro R, Mackem S, Zeller R. 2010. Distinct roles of Hand2 in initiating polarity and posterior Shh expression during the onset of mouse limb bud development. PLoS Genet 6:e1000901.

Gros J, Hu JK, Vinegoni C, Feruglio PF, Weissleder R, Tabin CJ. 2010. WNT5A/JNK and FGF/MAPK pathways regulate the cellular events shaping the vertebrate limb bud. Curr Biol 20:1993-2002.

Houzelstein D, Cohen A, Buckingham ME, Robert B. 1997. Insertional mutation of the mouse Msx1 homeobox gene by an nlacZ reporter gene. Mech. Dev. 65:123-133. 
Khokha MK, Hsu D, Brunet LJ, Dionne MS, Harland RM. 2003. Gremlin is the BMP antagonist required for maintenance of Shh and Fgf signals during limb patterning. Nat. Genet. 34:303-307.

Knudsen TB, Kochhar DM. 1981. The role of morphogenetic cell death during abnormal limb-bud outgrowth in mice heterozygous for the dominant mutation Hemimelia-extra toe (Hmx). J Embryol Exp Morphol 65 Suppl:289-307.

Krabbenhoft KM, Fallon JF. 1992. Talpid2 limb bud mesoderm does not express GHox-8 and has an altered expression pattern of GHox-7. Dev Dyn 194:52-62.

Kraus P, Fraidenraich D, Loomis CA. 2001. Some distal limb structures develop in mice lacking Sonic hedgehog signaling. Mech. Dev. 100:45-58.

Lallemand Y, Bensoussan V, Cloment CS, Robert B. 2009. Msx genes are important apoptosis effectors downstream of the Shh/Gli3 pathway in the limb. Dev Biol 331:189-198.

Lallemand Y, Nicola MA, Ramos C, Bach A, Cloment CS, Robert B. 2005. Analysis of Msx1; Msx2 double mutants reveals multiple roles for Msx genes in limb development. Development 132:3003-3014

Lettice LA, Heaney SJ, Purdie LA, Li L, de Beer P, Oostra BA, Goode D, Elgar G, Hill RE, de Graaff E. 2003. A long-range Shh enhancer regulates expression in the developing limb and fin and is associated with preaxial polydactyly. Hum Mol Genet 12:17251735 .

Logan M, Martin JF, Nagy A, Lobe C, Olson EN, Tabin CJ. 2002. Expression of Cre Recombinase in the developing mouse limb bud driven by a Prxl enhancer. Genesis 33:77-80.

Maatouk DM, Choi KS, Bouldin CM, Harfe BD. 2009. In the limb AER Bmp2 and Bmp4 are required for dorsal-ventral patterning and interdigital cell death but not limb outgrowth. Dev Biol 327:516-523.

Mao J, McGlinn E, Huang P, Tabin CJ, McMahon AP. 2009. Fgf-dependent Etv4/5 activity is required for posterior restriction of Sonic Hedgehog and promoting outgrowth of the vertebrate limb. Dev Cell 16:600-606.

Marazzi G, Wang Y, Sassoon D. 1997. Msx2 is a transcriptional regulator in the BMP4mediated programmed dell-death pathway. Dev. Biol. 186:127-138.

Michaud JL, Lapointe F, Le Douarin NM. 1997. The dorsoventral polarity of the presumptive limb is determined by signals produced by the somites and by the lateral somatopleure. Development 124:1453-1463.

Michos O, Panman L, Vintersten K, Beier K, Zeller R, Zuniga A. 2004. Gremlin-mediated BMP antagonism induces the epithelial-mesenchymal feedback signaling controlling metanephric kidney and limb organogenesis. Development 131:3401-3410.

Nakatomi M, Wang XP, Key D, Lund JJ, Turbe-Doan A, Kist R, Aw A, Chen Y, Maas RL, Peters H. 2010. Genetic interactions between Pax9 and Msx1 regulate lip development and several stages of tooth morphogenesis. Dev Biol 340:438-449.

Neubuser A, Koseki H, Balling R. 1995. Characterization and developmental expression of Pax9, a paired-box-containing gene related to Pax1. Dev Biol 170:701-716.

Ogawa T, Kapadia H, Feng JQ, Raghow R, Peters H, D'Souza RN. 2006. Functional consequences of interactions between Pax9 and Msx1 genes in normal and abnormal tooth development. J Biol Chem 281:18363-18369.

Ovchinnikov DA, Selever J, Wang Y, Chen YT, Mishina Y, Martin JF, Behringer RR. 2006. BMP receptor type IA in limb bud mesenchyme regulates distal outgrowth and patterning. Dev. Biol. 295:103-115. 
Peters H, Neubuser A, Kratochwil K, Balling R. 1998. Pax9-deficient mice lack pharyngeal pouch derivatives and teeth and exhibit craniofacial and limb abnormalities. Genes Dev 12:2735-2747.

Pizette S, Niswander L. 1999. BMPs negatively regulate structure and function of the limb apical ectodermal ridge. Development 126:883-894.

Robert B. 2007. Bone morphogenetic protein signaling in limb outgrowth and patterning. Dev Growth Differ 49:455-468.

Sagai T, Masuya H, Tamura M, Shimizu K, Yada Y, Wakana S, Gondo Y, Noda T, Shiroishi T. 2004. Phylogenetic conservation of a limb-specific, cis-acting regulator of Sonic hedgehog ( Shh). Mamm Genome 15:23-34.

Sanz-Ezquerro JJ, Tickle C. 2003. Fgf signaling controls the number of phalanges and tip formation in developing digits. Curr Biol 13:1830-1836.

Satokata I, Ma L, Ohshima H, Bei M, Woo I, Nishizawa K, Maeda T, Takano Y, Uchiyama M, Heaney S, Peters H, Tang Z, Maxson R, Maas R. 2000. Msx2 deficiency in mice causes pleiotropic defects in bone growth and ectodermal organ formation. Nat Genet 24:391-395.

Satokata I, Maas R. 1994. Msx1 deficient mice exhibit cleft palate and abnormalities of craniofacial and tooth development. Nat Genet 6:348-356.

Selever J, Liu W, Lu MF, Behringer RR, Martin JF. 2004. Bmp4 in limb bud mesoderm regulates digit pattern by controlling AER development. Dev. Biol. 276:268-279.

Sharpe J, Lettice L, Hecksher-Sorensen J, Fox M, Hill R, Krumlauf R. 1999. Identification of sonic hedgehog as a candidate gene responsible for the polydactylous mouse mutant Sasquatch. Curr Biol 9:97-100.

Sun X, Lewandoski M, Meyers EN, Liu YH, Maxson RE, Jr., Martin GR. 2000. Conditional inactivation of Fgf4 reveals complexity of signalling during limb bud development. Nat Genet 25:83-86.

Wang B, Fallon JF, Beachy PA. 2000. Hedgehog-regulated processing of Gli3 produces an anterior/posterior repressor gradient in the developing vertebrate limb. Cell 100:423434.

Wang CK, Omi M, Ferrari D, Cheng HC, Lizarraga G, Chin HJ, Upholt WB, Dealy CN, Kosher RA. 2004. Function of BMPs in the apical ectoderm of the developing mouse limb. Dev Biol 269:109-122.

Wyngaarden LA, Vogeli KM, Ciruna BG, Wells M, Hadjantonakis AK, Hopyan S. 2010. Oriented cell motility and division underlie early limb bud morphogenesis. Development 137:2551-2558.

Xu X, Weinstein M, Li C, Naski M, Cohen RI, Ornitz DM, Leder P, Deng C. 1998. Fibroblast growth factor receptor 2 (FGFR2)-mediated reciprocal regulation loop between FGF8 and FGF10 is essential for limb induction. Development 125:753-765.

Zhang Z, Song Y, Zhao X, Zhang X, Fermin C, Chen Y. 2002. Rescue of cleft palate in Msx1-deficient mice by transgenic Bmp4 reveals a network of BMP and Shh signaling in the regulation of mammalian palatogenesis. Development 129:4135-4146.

Zhang Z, Sui P, Dong A, Hassell J, Cserjesi P, Chen YT, Behringer RR, Sun X. 2010. Preaxial polydactyly: interactions among ETV, TWIST1 and HAND2 control anteriorposterior patterning of the limb. Development 137:3417-3426.

Zhang Z, Verheyden JM, Hassell JA, Sun X. 2009. FGF-regulated Etv genes are essential for repressing Shh expression in mouse limb buds. Dev Cell 16:607-613.

Zuniga A, Haramis AP, McMahon AP, Zeller R. 1999. Signal relay by BMP antagonism controls the SHH/FGF4 feedback loop in vertebrate limb buds. Nature 401:598-602. 


\section{FIGURE LEGENDS}

\section{Fig. 1. Limb phenotype of the $M s x 1^{\text {null/null }} M s x 2^{+/ n u l l}$ mutant}

(A-H') In situ hybridization (ISH) on fore- and hindlimbs of wild-type and Msx $I^{\text {null/null }}$ $M s x 2^{+/ n u l l}$ mutant embryos using Hoxd11, Hoxd12 (at $12.5 \mathrm{dpc}$ ) or Pax9 and Dlx5 (at 11.5 dpc) probes. Only minor differences are noticeable in forelimbs (E,E') whereas, in the compound mutant hindlimbs, posterior markers (Hoxd11 and Hoxd12) clearly expand anteriorly (arrowheads in B' and D' respectively), and anterior markers (Pax9 and Dlx5) are dramatically downregulated anteriorly (F',H'). (I, I') Forelimb skeletal preparations stained with alizarin red (bone) and alcian blue (cartilage) from a wild type (I) and an MsxI $I^{\text {null/null }}$ $M s x 2^{+/ n u l l}$ (I') embryo at $18.5 \mathrm{dpc}$. The thumb (T) and the falciform bone (F), that are normally separated, are partially fused in the mutant. (J-L') ISH on hindlimbs at $11.5 \mathrm{dpc}$, using Gli1, Ptcl and Gli3 probes. No difference is detectable between wild-type and Msx $1^{\text {null/null }} M s \times 2^{+/ n u l l}$ mutant littermates.

Fig. 2. AER formation in the Prx1-Cre Msx $1^{\text {null/null }} M s x 2^{\text {null/Flox }}$ conditional double mutant

(A-D) AER morphology revealed by $F g f 8$ expression at $11.5 \mathrm{dpc}$ in forelimbs of a wild-type (A), a Prxl-Cre Msx $1^{\text {null/null }} M s x 2^{\text {null/Flox }}$ conditional double mutant (B), an Msx $I^{\text {null/null }}$ $M s x 2^{\text {null/null }}$ double null mutant $(\mathbf{C})$ and an Msx $1^{\text {null/null }} M s x 2^{+/ n u l l}$ compound mutant (D) embryo. All panels display forelimbs and are orientated with the anterior to the left. In all panels, arrowheads indicate AER boundaries. Note that in the Msx $1^{\text {null/null }} M s x 2^{\text {null/null }}$ double null mutant $(\mathbf{C})$, the AER is truncated anteriorly. In the Prxl-Cre Msx $1^{\text {null/null }} M$ Msx $2^{\text {null/Flox }}$ 
conditional double mutant (B), the AER slightly extends anteriorly as compared to the control (A). This difference is not due to the low Msx genetic dosage in the AER (only one Msx2 functional allele) as such a phenotype is not detectable in the $M s x 1^{\text {null/null }} M s \times 2^{+/ n u l l}$ compound mutant (D).

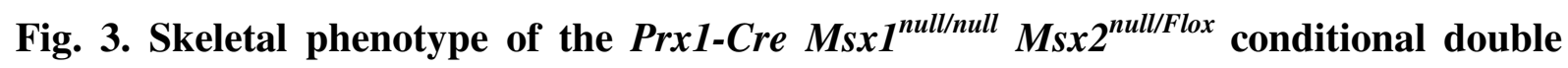
mutant

(A-H) Alizarin red/alcian blue-stained skeletal preparations of control (A,C,E,G) and PrxlCre Msxi $1^{\text {null/null }} M s x 2^{\text {null/Flox }}$ conditional double mutant $(\mathbf{B , D , F , H ) ~ n e w - b o r n ~ a n i m a l s . ~}$ Mesenchyme specific Msx inactivation leads to severe defects along the anteroposterior axis in the forelimb $(\mathbf{A}, \mathbf{C}, \mathbf{E}, \mathbf{F})$, and to a much lesser extent in the hindlimb $(\mathbf{B}, \mathbf{D}, \mathbf{G}, \mathbf{H})$. $(\mathbf{A}, \mathbf{C})$ In the forelimb, the deltoid tuberosity (arrow in A) is missing in the stylopod of the conditional double mutant, while in the zeugopod, the radius (R) is either shorter (C) or absent (Supplementary Fig. S2). The autopod displays a preaxial polydactyly. (B,D) In the hindlimb, the phenotype is less striking. The stylopod is normal and the zeugopod affected in only one third of the cases (Supplementary Fig. S2). The polydactyly is generally limited to the presence of a small anterior cartilaginous formation (arrow in D). (E-H) Details of the autopod phenotype. In all panels anterior is to the left, digits are numbered from anterior (1) to posterior (5). Phalanges (p1-p3) are delimited by brackets. Note that in both fore- $(\mathrm{E}, \mathrm{F})$ and hindlimbs $(\mathrm{G}, \mathrm{H})$, the phalanges of the mutants $(\mathrm{F}, \mathrm{H})$ are not ossified and that the distal phalanges (P3, occasionally P2) are either absent or ill-developed without signs of chondrification. (I-L) Fore- (I,J) and hindlimb (K,L) autopods of control (I,K) and mutant $(\mathrm{J}, \mathrm{L})$ new-born animals, before skeletal preparation. Note that the nails visible in the control animals (arrows in I and K) are absent in the mutants, in both fore- and hindlimbs. 
Fig. 4. Abnormal anteroposterior polarity in the limb bud of the Prx1-Cre Msxi ${ }^{\text {null/null }}$ $M s x 2^{\text {null/Flox }}$ conditional double mutant

Limb bud expression patterns of Hoxd12 (A-D), Hoxd11 (E-F), Hand2 (G-H) and Pax9 (I-J)

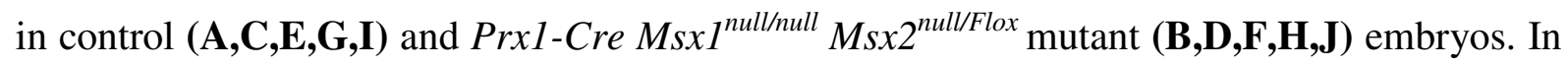
all panels, anterior is to the left. Panels A,B,E and F display forelimb buds at $12.5 \mathrm{dpc}$, panels C,D,G,H,I and $\mathbf{J}$ display forelimb buds at $11.5 \mathrm{dpc}$ (as indicated on the left of the panels). Note anterior ectopic expression of the genes normally expressed solely in the posterior region (Hoxd12, Hoxd11, Hand2). Pax9 is normally expressed only in digit 1 presumptive territory (I). Its expression is almost undetectable in the mutant limb bud, except for a small intermediate region (arrow in $\mathrm{J}$ ). This region is negative for Hoxd12, Hoxd11 and Hand2 expression in the mutant (arrows in B, D, F and $\mathrm{H}$ respectively).

Fig. 5. Ectopic Shh signaling activity at the anterior border of the Prx1-Cre Msx1 ${ }^{\text {null/null }}$ $M s x 2^{\text {null/Flox }}$ mutant forelimb bud is not correlated with Etv gene dowregulation. Shh $(\mathbf{A}, \mathbf{B})$, Gli1 (C,D), Etv4 $(\mathbf{E}, \mathbf{F})$ and Etv5 $(\mathbf{G}, \mathbf{H})$ expression in control $(\mathbf{A}, \mathbf{C}, \mathbf{E}, \mathbf{G})$ and conditional mutant (B,D,F,H) embryos at 11.5 dpc. (A-D) Anterior ectopic expression of Shh and Glil is indicated with a black arrowhead in (B) and (D) respectively. (E-H) The brackets delimited the anterior domain where Etv genes are normally at a weaker level in control limb buds and upregulated in the mutants.

\section{Fig. 6. Anteroposterior polarity modification in the Msx conditional double mutant} forelimb buds at $10.75 \mathrm{dpc}$

(A-C) Shh and Fgf4 expression at $10.75 \mathrm{dpc}$ in a control (A), an Msx conditional double mutant (B) and a $S h h^{H x /+}$ mutant (C) embryo. At this stage, Shh expression is similar in the control and the two kinds of mutants, whereas Fgf4 domain already extends anteriorly in the 
AER of the Prxl-Cre Msxillinull Msx $2^{\text {null/Flox }}$ conditional double mutant. (D-F) Hand2 and Fgf4 expression at $10.75 \mathrm{dpc}$ in a control (D), an Msx conditional double mutant (E) and a $M s x 1^{\text {null/null }} M s x 2^{+/ n u l l}$ compound mutant (F) embryo. At this stage, compared to the control embryo (D), Fgf4 expression extends anteriorly in the Prxl-Cre Msx1 $1^{\text {null/null }}$ Msx $2^{\text {null/Flox }}$ conditional double mutant embryo (E) but not in the Msx $1^{\text {null/null }} M s \times 2^{+/ n u l l}$ compound mutant (F). Conversely, in the mesenchyme, Hand2 expression is similar in the three genotypes.

\section{Fig. 7. Global BMP signal modification in the Msx conditional double mutant forelimb} buds

Bmp4 (A-C), Bmp2 (D-F), Bmp7 (G-I and M-O) and Gremlin (J-L and P-R) expression in control (A,D,G,J,M,P), Msx conditional double mutant $(\mathbf{B}, \mathbf{E}, \mathbf{H}, \mathbf{K}, \mathbf{N}, \mathbf{Q})$ and $S h h^{H x /+}$ mutant (C,F,I,L,O,R) embryos, at $11.5 \mathrm{dpc}(\mathbf{A}-\mathbf{L})$ or $10.75 \mathrm{dpc}(\mathbf{M}-\mathbf{R})$.

(A-C) At $11.5 \mathrm{dpc}$, Bmp4 expression is downregulated anteriorly in the Prxl-Cre Msx $1^{\text {null/null }}$ $M s x 2^{\text {null/Flox }}$ conditional double mutant $(\mathbf{B})$ compare to the control (A), but not in the $S h h^{H x /+}$ mutant (C). (D-F) Bmp2 expression at $11.5 \mathrm{dpc}$. In $(\mathbf{E})$ and $(\mathbf{F})$ the arrowheads point to the small ectopic anterior expression domain visible in the AER. (G-I) Bmp7 expression at 11.5 dpc. In the three panels, the rectangles delimitate the anteriormost region of the limb bud where Bmp7 is slightly upregulated in both the Prx1-Cre Msx $1^{\text {null/null }} M s x 2^{\text {null/Flox }}$ conditional double mutant $(\mathbf{H})$ and the $S h h^{H x /+}$ mutant (I) embryo compared to the control embryo $(\mathbf{G})$. (J-L) Gremlin expression at $11.5 \mathrm{dpc}$. In both the Prxl-Cre Msx $1^{\text {null/null }}$ Msx $2^{\text {null/Flox }}$ conditional double mutant $(\mathbf{K})$ and the $S h h^{H x /+}$ mutant $(\mathbf{L})$ embryos, a clear ectopic expression domain is visible in the anterior mesenchyme. (M-O) Bmp7 expression at $10.75 \mathrm{dpc}$. As for the 11.5 dpc stage, $B m p 7$ expression is slightly upregulated anteriorly in the Msx conditional double mutant $(\mathbf{N})$ and the $S h h^{H x /+}$ mutant (O) embryos (compare the region delimitated by the rectangle in $\mathrm{M}, \mathrm{N}$ and $\mathrm{O})$. (P-R) Gremlin expression at $10.75 \mathrm{dpc}$. At this stage, Gremlin 
is ectopically expressed in the anteriormost region of the mesenchyme in the Msx conditional double mutant $(\mathbf{Q})$ and the $S h h^{H x /+}$ mutant $(\mathbf{R})$ embryos (compare the region delimitated by the rectangle in $\mathrm{P}, \mathrm{Q}$ and $\mathrm{R})$.

\section{Fig. 8. Apoptosis and cell proliferation at $\mathbf{1 1 . 5}$ dpc}

$(\mathbf{A}, \mathbf{B})$ At $11.5 \mathrm{dpc}$, active Caspase-3 analysis reveals that the anterior cell death domain normally observed in wild-type embryos at this stage (rectangle in $\mathbf{A}$ ), is undetectable in the Msx conditional double mutant (B). (C,D) At $11.5 \mathrm{dpc}$, phospho-histone H3 analysis reveals an homogenous and high level of cell proliferation in the limb bud mesenchyme of both the control (C) and the Msx conditional double mutant (D) embryos 


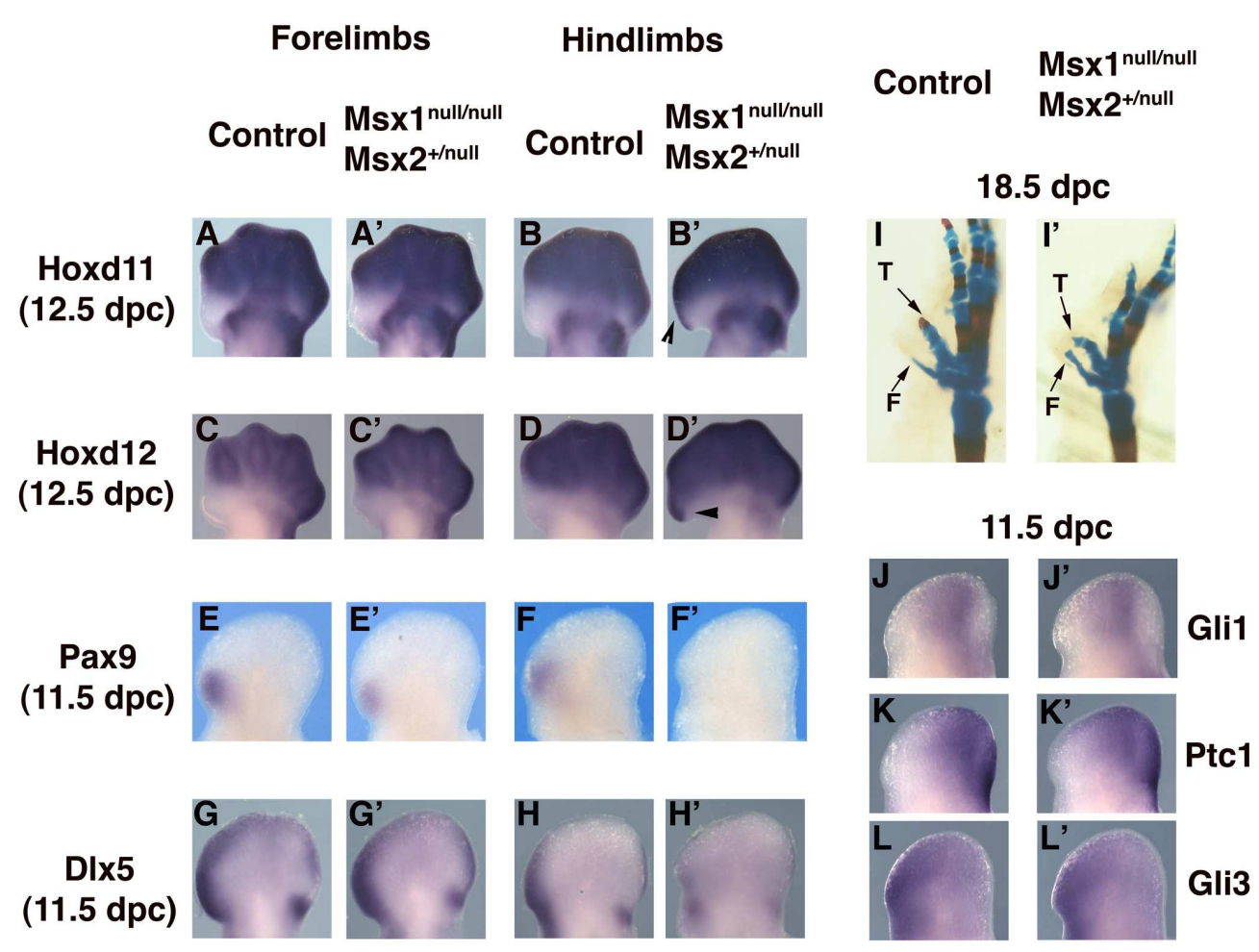

Figure 1

$175 \times 130 \mathrm{~mm}(300 \times 300$ DPI $)$ 


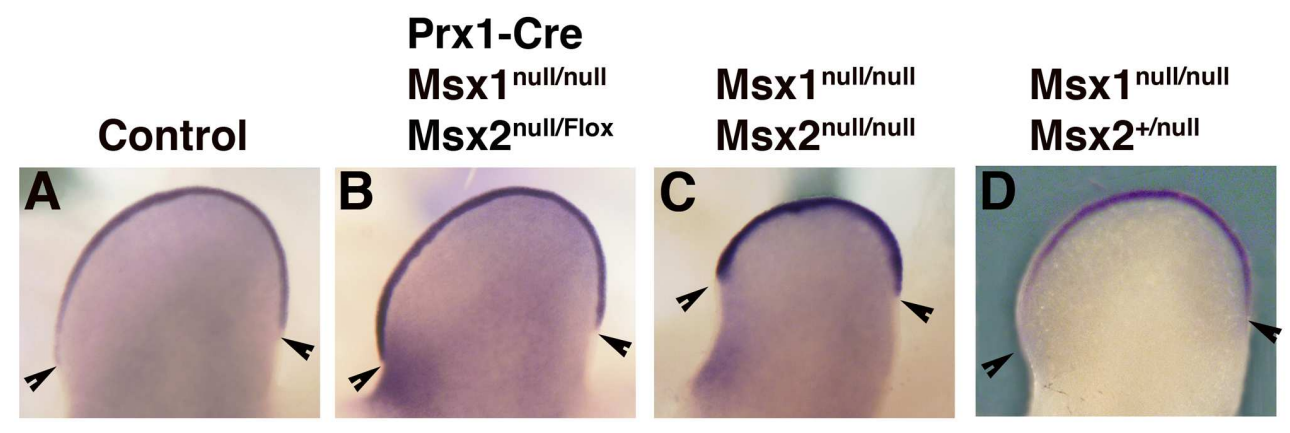

Figure 2

$175 \times 60 \mathrm{~mm}(300 \times 300$ DPI $)$

John Wiley \& Sons, Inc. 
Forelimbs

A

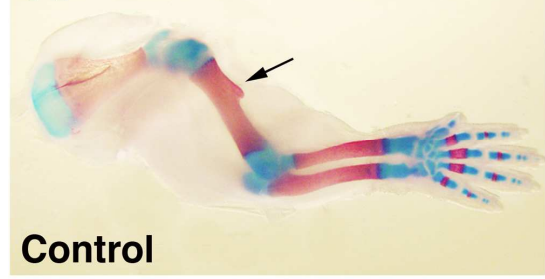

C

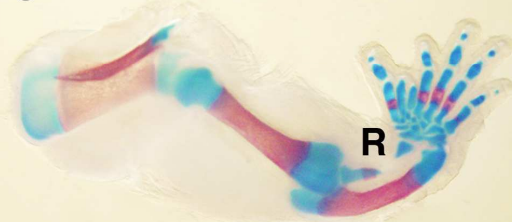

Prx1-Cre Msx $1^{\text {null/null } M s x 2^{\text {null/Flox }}}$

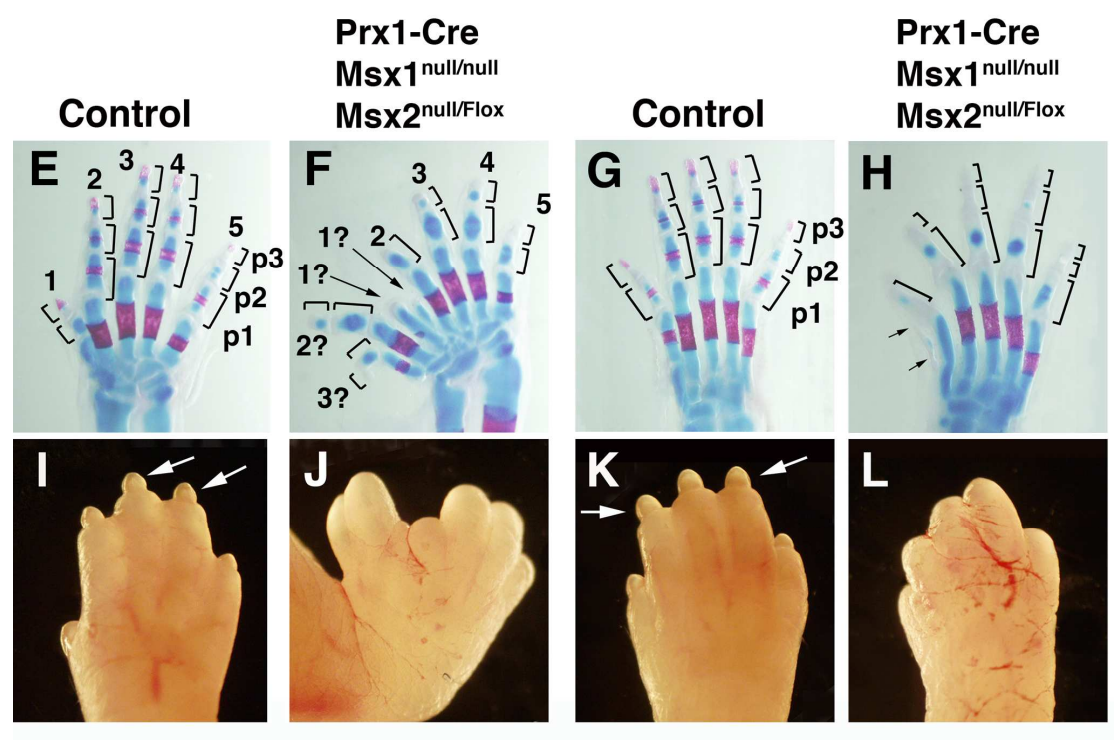

Figure 3

$175 \times 245 \mathrm{~mm}(300 \times 300$ DPI $)$

John Wiley \& Sons, Inc. 


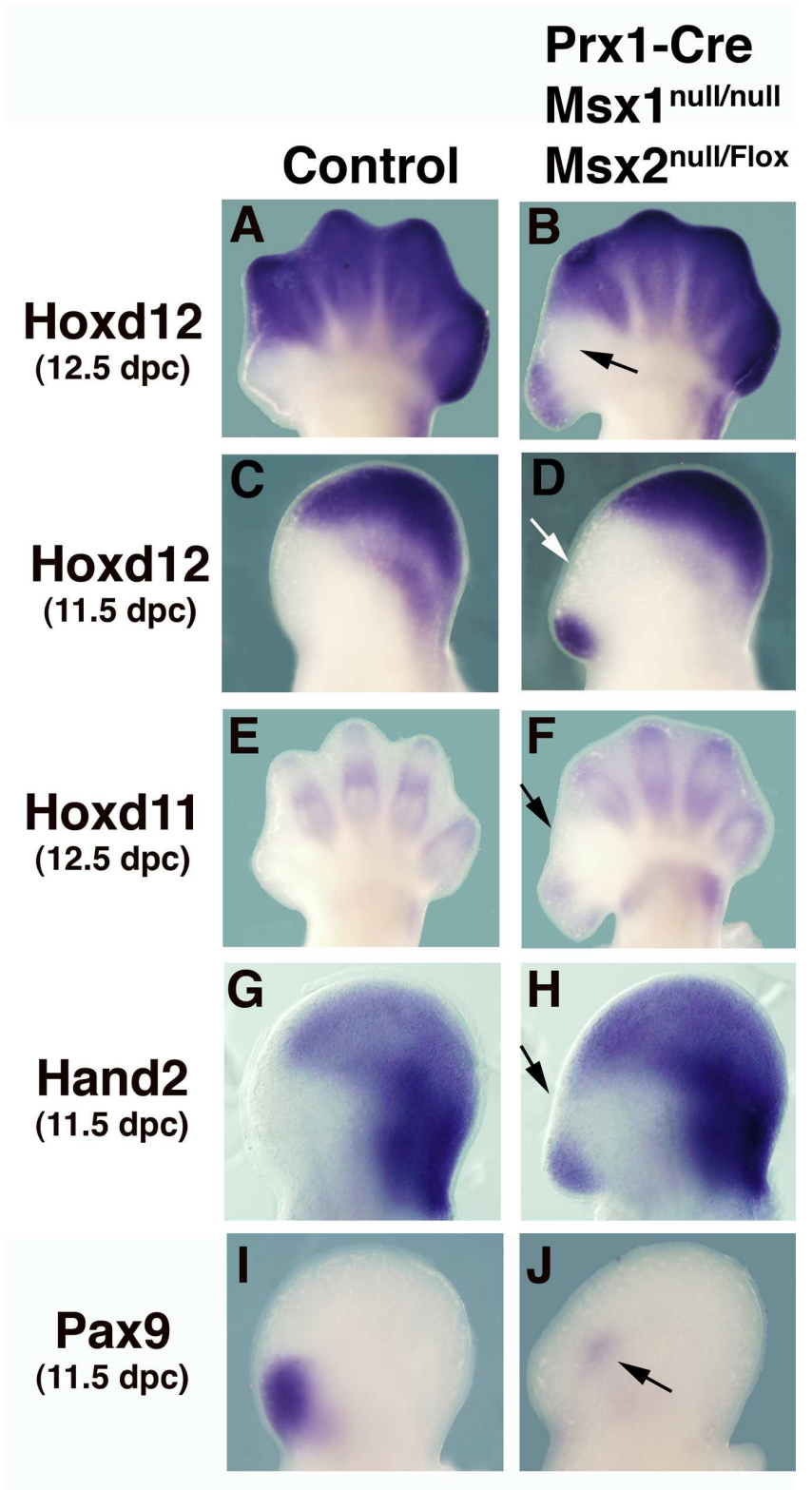

Figure 4

$114 \times 215 \mathrm{~mm}(300 \times 300$ DPI $)$

John Wiley \& Sons, Inc. 


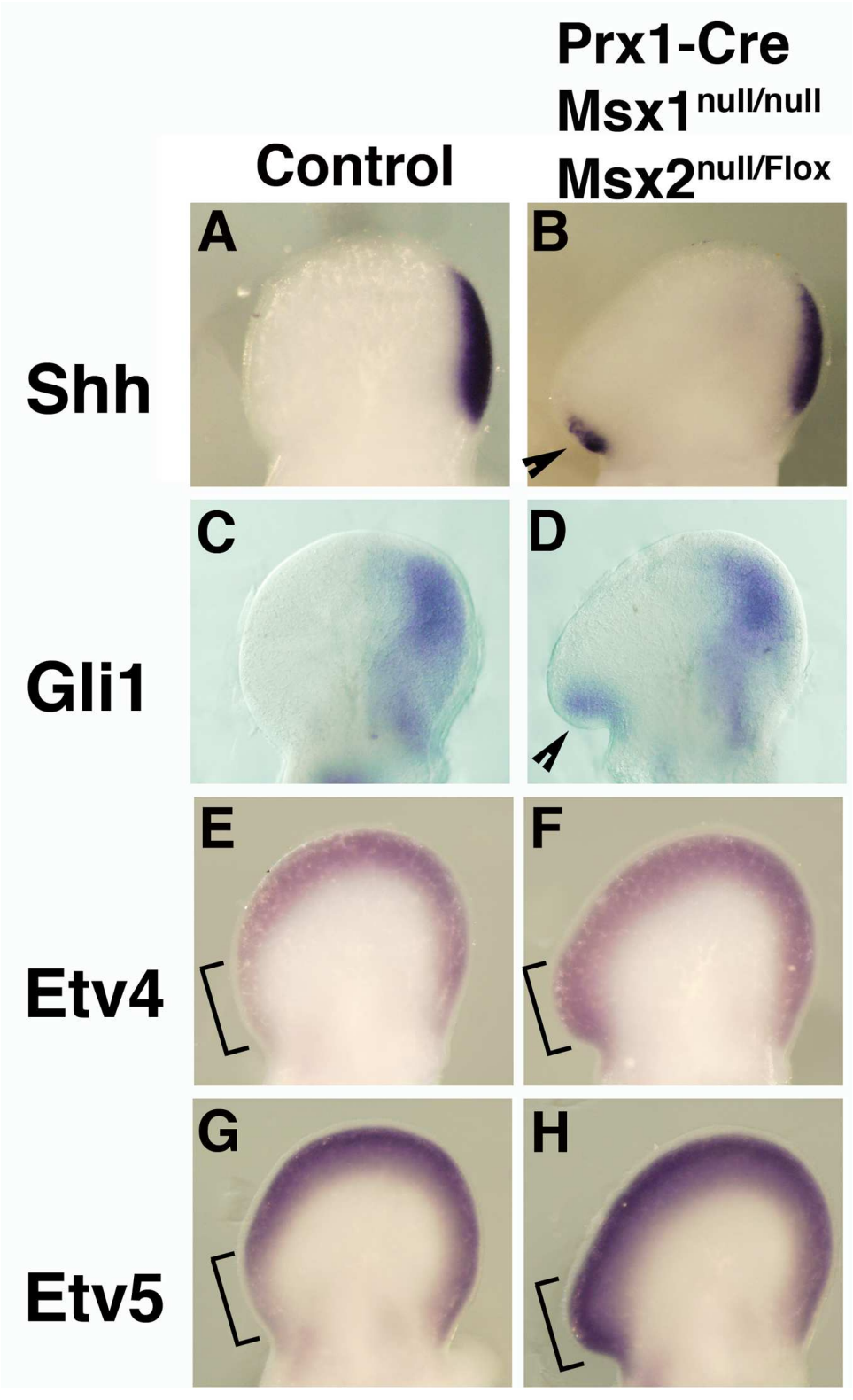

$114 \times 186 \mathrm{~mm}(300 \times 300 \mathrm{DPI})$

John Wiley \& Sons, Inc. 


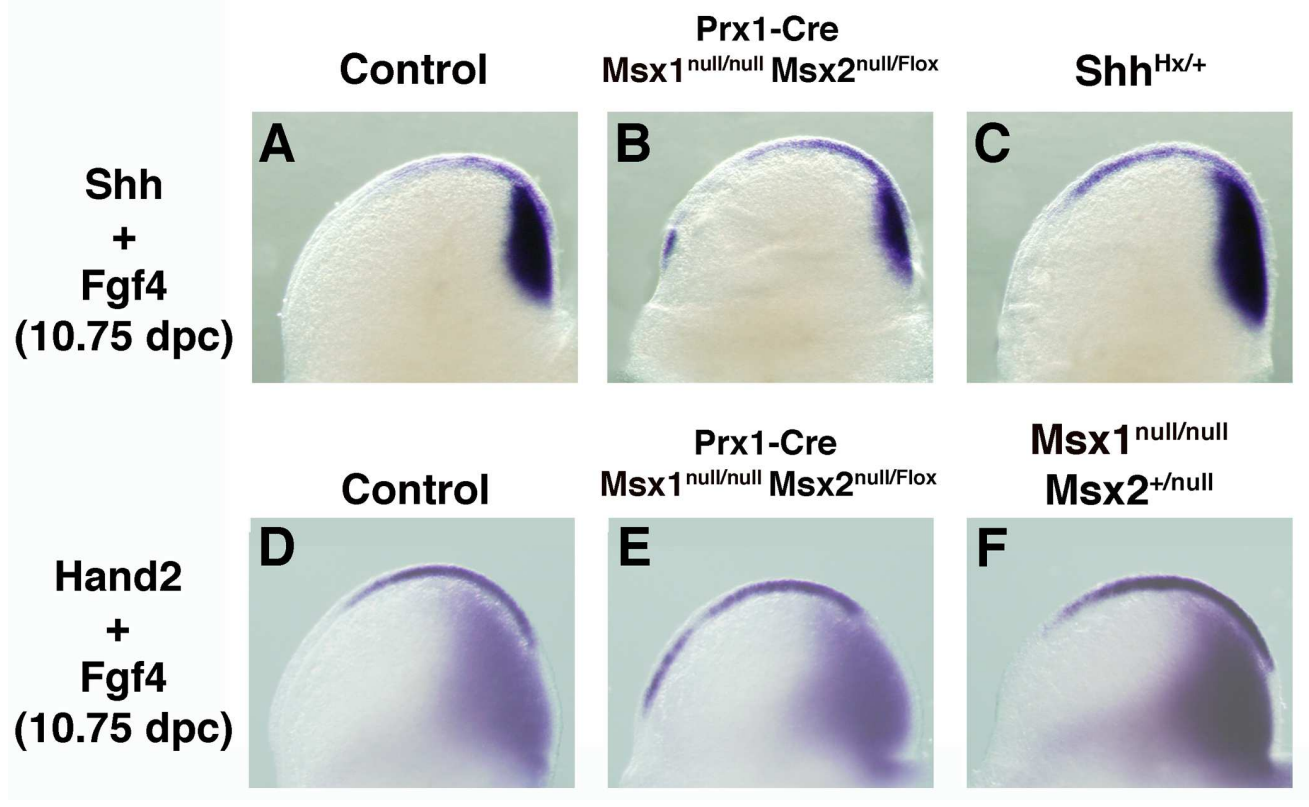

Figure 6

$175 \times 109 \mathrm{~mm}(300 \times 300$ DPI $)$ 


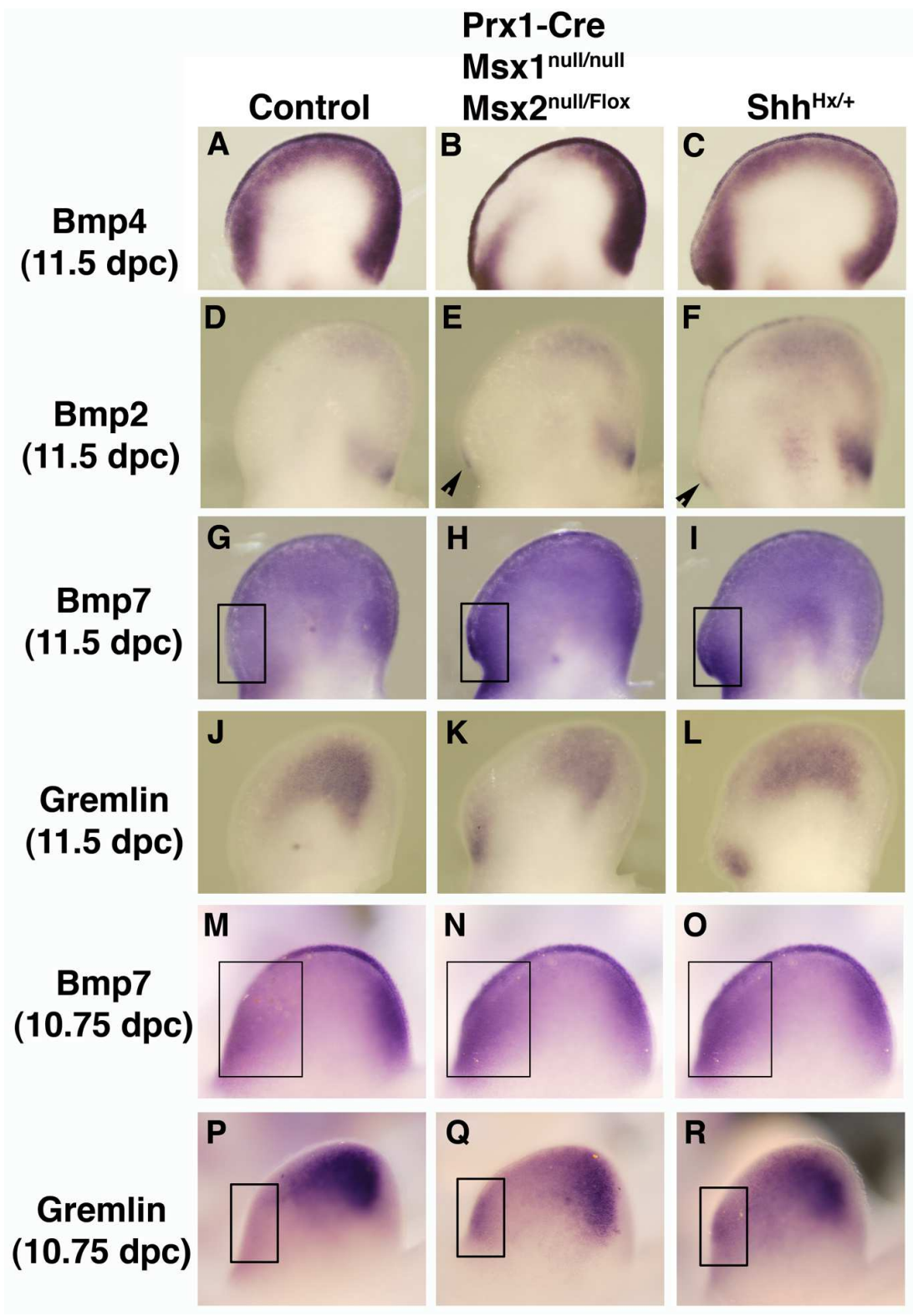

Figure 7

$114 \times 164 \mathrm{~mm}(300 \times 300 \mathrm{DPI})$ 


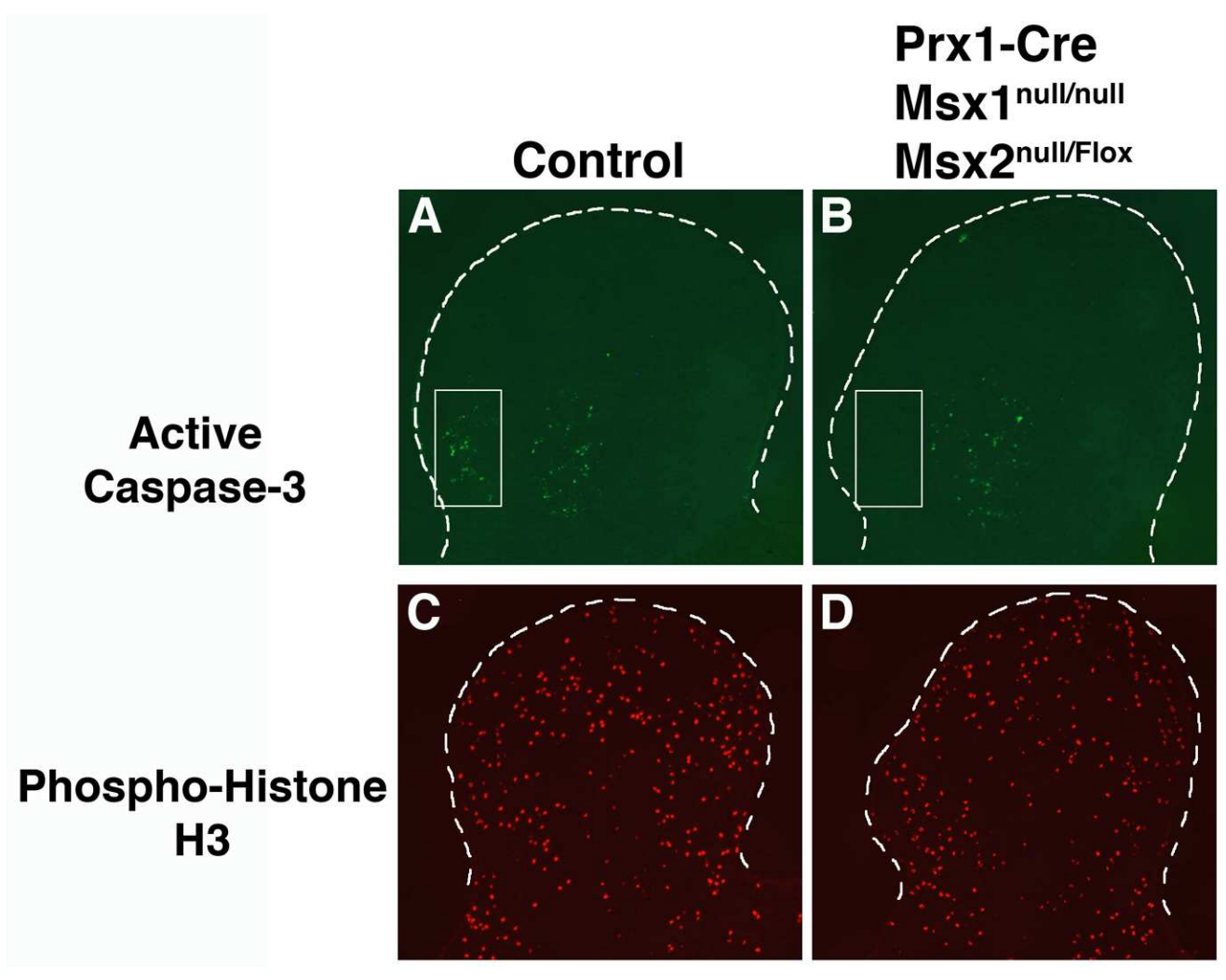

Figure 8

$114 \times 90 \mathrm{~mm}(300 \times 300 \mathrm{DPI})$

John Wiley \& Sons, Inc. 